\title{
Effects of oily fish intake on cardiovascular risk markers, cognitive function, and behavior in school-aged children: study protocol for a randomized controlled trial
}

Camilla T. Damsgaard ${ }^{1 *}$, Lotte Lauritzen ${ }^{1}$, Hanne Hauger ${ }^{1}$, Stine Vuholm¹, Marie N. Teisen ${ }^{1}$, Christian Ritz ${ }^{1}$, Max Hansen², Janni Niclasen ${ }^{3,4}$ and Christian Mølgaard ${ }^{1}$

\begin{abstract}
Background: Most children in Western populations do not meet recommendations for fish consumption. Oily fish is an important source of n-3 long-chain polyunsaturated fatty acids (LCPUFA), which reduce blood pressure and plasma triacylglycerol in adults and may affect cognitive development and behavior. However, to our knowledge, the potential effects of oily fish on cardiometabolic health, cognitive function, and behavior in children have not been investigated. The aim of the FiSK Junior study is to investigate the effects of oily fish consumption on cardiovascular risk markers, cognitive function, and behavior in healthy children.
\end{abstract}

Methods/design: We are conducting a randomized controlled trial with 8- to 9-year-old Danish children, comparing the effect of consuming $300 \mathrm{~g} /$ week of oily fish with poultry (control) for 12 weeks between August 2016 and June 2017. The primary outcomes are blood pressure and fasting plasma triacylglycerol, which will be measured at baseline and endpoint. In addition, we will assess erythrocyte fatty acid composition (compliance), heart rate, plasma cholesterol, markers of glucose homeostasis, growth and body composition, dietary intake, and physical activity and sleep. We will also examine effects on cognitive function (attention, memory, and executive functions) by using standardized tests, behavior and emotions by administering parent-rated questionnaires and child interviews, and we will measure physiological stress response and cortisol levels. We need 150 children to complete the trial to detect a between-groups difference of $2.7 \mathrm{mmHg}$ in diastolic blood pressure and $0.13 \mathrm{mmol} / \mathrm{L}$ in plasma triacylglycerol; thus, we aim to recruit 200 children. All outcomes will be analyzed in completer analysis supplemented with sensitivity analyses for the primary outcomes, and attention will be given to potential sex and genotype specificity.

Discussion: The results of the FiSK Junior study are expected to fill important gaps in the current knowledge about the importance of dietary fish and n-3 LCPUFA for children's health and development, and may be used when setting dietary recommendations.

Trial registration: ClinicalTrials.gov identifier: NCT02809508. Registered on 22 June 2016.

Keywords: n-3 PUFA, Child health, Dietary recommendations, Cognitive function, Cardiovascular risk factors, Mental health

\footnotetext{
* Correspondence: ctd@nexs.ku.dk

${ }^{1}$ Department of Nutrition, Exercise and Sports, Faculty of Science, University

of Copenhagen, Rolighedsvej 26, 1958 Frederiksberg C, Denmark

Full list of author information is available at the end of the article
} 


\section{Background}

Fish is an important dietary source of iodine, selenium, zinc, and high-quality proteins. Oily fish is also the main dietary source of $n-3$ long-chain polyunsaturated fatty acids (LCPUFA), which have been shown to provide protection from cardiovascular disease [1] and may affect cognitive function and behavior. Most Western countries recommend two to four servings of fish weekly, typically half thereof as oily fish, but especially children do not meet these recommendations [2-4]. Danes are recommended to consume $350 \mathrm{~g} /$ week of fish, whereof $200 \mathrm{~g} /$ week should be oily fish such as salmon, mackerel, and herring [5], but less than $10 \%$ of Danish children aged 4 to 9 years reach this intake [3]. Oily fish is also an important natural source of vitamin D, of which most Danes consume too little [3]. Vitamin D deficiency is common at northern latitudes during winter, when the contribution of vitamin D from sun exposure is negligible [6]. Low vitamin D status can impair children's bone health [7] and may also affect immune function and cardiovascular health [8].

Fish consumption is associated with reduced cardiovascular mortality in adults [9]. This is presumed to be due mainly to the content of n-3 LCPUFA, as these fatty acids (typically administered as fish oil) are well known to reduce cardiovascular risk factors, especially blood pressure [10], heart rate [11], and plasma triacylglycerol [12] in adults. Our previous randomized trials with fish oil provided to infants and adolescents indicated that n3 LCPUFA have comparable effects in children [13-15]. Randomized controlled trials of oily fish in adults rather than fish oils have shown mixed results, but most indicate that consumption of oily fish lowers blood pressure and plasma triacylglycerol (see, e.g., $[16,17])$. To our knowledge, no randomized trial has investigated the effect of fish consumption per se on cardiovascular health in children.

During brain growth, n-3 LCPUFA, specifically docosahexaenoic acid (DHA, 22:6-n-3), are incorporated at uniquely high levels in the brain [18], which may confer increased needs for these fatty acids in childhood. The rate of incorporation has been shown to be dietdependent and is believed to play a role in the cognitive development of the child [18]. Most trials investigating the effects of $n-3$ LCPUFA on cognitive function have been conducted with formula-fed infants or with infants of mothers receiving fish oil. Meta-analyses of these studies have not shown pronounced benefits of $n-3$ LCPUFA on neurodevelopmental outcomes during the first 2 years of life [19]. However, this may be because the effect of n-3 LCPUFA is sex-dependent [20, 21]. Few studies of young children have examined the effects on specific cognitive functions, and with conflicting results, possibly due to methodological limitations [22]. In addition to cognitive function, studies investigating the effects of n-3 LCPUFA on behavior and emotional outcomes are sparse. A few randomized trials in healthy schoolchildren have shown that children supplemented with fish oil had reduced impulsivity, antisocial behavior, and physical activity during school hours [23-25], but this needs more investigation.

Most studies of fish and n-3 LCPUFA have been conducted with males or without specific attention to sex. However, recent work indicates that some effects of n-3 LCPUFA in children may be sex-specific, especially in relation to blood pressure and cognitive function [26, 27]. Moreover, studies indicate that the effects of n-3 LCPUFA, and therefore potentially also oily fish intake, are genotype-dependent [28].

\section{Aim}

In the FiSK (Fish, children, health, and cognition (Fisk, børn, Sundhed og Kognition)) Junior study, we will investigate whether intake of oily fish compared with poultry affects cardiovascular risk markers, primarily blood pressure and plasma triacylglycerol, in children. Moreover, we will assess the effects on cognitive function, behavior, and emotions and give special attention to potential sex and genotype specificity. This paper presents the rationale, design, and potential implications of the study.

\section{Methods \\ Study design and ethics}

The FiSK Junior study is a randomized controlled parallel trial in which Danish children will receive $300 \mathrm{~g} /$ week of oily fish or poultry (control) for $12 \pm 3$ weeks between August 2016 and June 2017. Measurements and biological sampling will be performed at baseline and endpoint at the Department of Nutrition, Exercise and Sports (NEXS), University of Copenhagen, Frederiksberg, Denmark (Fig. 1). See Additional file 1 for an overview of the Standard Protocol Items: Recommendations for Interventional Trials (SPIRIT) 2013 checklist items.

The study protocol has been approved by the Committee on Biomedical Research Ethics for the Capital Region of Denmark (H-16018225) and was registered with ClinicalTrials.gov (NCT02809508) on 22 June 2016. The study will be conducted in accordance with the guidelines in the Declaration of Helsinki, and informed written consent will be obtained from all custody holders of the children. Also, we will follow the Danish Act on Processing of Personal Data for all data collected in the study.

\section{Participants}

Participants will be healthy 8- to 9-year-old children living in the Capital Region of Denmark. Children must speak Danish, like oily fish and chicken, not consume oily fish more than once per week, and must not take 


\begin{tabular}{|c|c|c|c|c|c|c|c|c|c|c|c|}
\hline Timepoint (weeks) & $\begin{array}{l}\text { Recruitment } \\
-5 \pm 3\end{array}$ & $\begin{array}{c}\text { Pre-visit } \\
-3 \pm 2\end{array}$ & $\begin{array}{l}\text { At home } \\
-2 \pm 2\end{array}$ & $\begin{array}{c}\text { Baseline } \\
0\end{array}$ & $2 \pm 1$ & $4 \pm 1$ & $6 \pm 1$ & $8 \pm 1$ & $10 \pm 1$ & $\begin{array}{c}\text { At home } \\
11 \pm 3\end{array}$ & $\begin{array}{c}\text { Endpoint } \\
12 \pm 3\end{array}$ \\
\hline \multicolumn{12}{|l|}{ Enrollment } \\
\hline Eligibility screening & $\mathbf{x}$ & & & & & & & & & & \\
\hline Information meeting & $\mathrm{x}$ & & & & & & & & & & \\
\hline Informed consent & $x$ & & & & & & & & & & \\
\hline Instructions & & $\mathbf{x}$ & & & & & & & & & \\
\hline Cognitive pre-testing & & $\mathbf{x}$ & & & & & & & & & \\
\hline Allocation & & & & $\mathbf{x}$ & & & & & & & \\
\hline \multicolumn{12}{|l|}{ Interventions } \\
\hline \multicolumn{12}{|l|}{ Eat oily fish (intervention) } \\
\hline \multicolumn{12}{|l|}{ Eat poultry (control) } \\
\hline Collect study foods & & & & $\mathbf{x}$ & $\mathbf{x}$ & $\mathbf{x}$ & $\mathbf{x}$ & $\mathbf{x}$ & $\mathbf{x}$ & & \\
\hline \multicolumn{12}{|l|}{ Assessments } \\
\hline Sociodemo graphics & & & & $\mathbf{X}$ & & & & & & & \\
\hline Dietary intake & & & $\mathbf{x}$ & & & & & & & $\mathbf{x}$ & \\
\hline Physical activity and sleep & & & $x$ & & & & & & & $x$ & \\
\hline Anthropometry & & & & $\mathbf{x}$ & & & & & & & $\mathbf{x}$ \\
\hline Body composition & & & & $\mathbf{x}$ & & & & & & & $\mathbf{x}$ \\
\hline Blood pressure and HR & & & & $x$ & & & & & & & $x$ \\
\hline 4-hour ECG & & & & $x$ & & & & & & & $\ddot{x}$ \\
\hline Biological samples & & & & $\mathbf{x}$ & & & & & & & $\mathbf{x}$ \\
\hline Cognitive function and behar & aviour & & & $x$ & & & & & & & $x$ \\
\hline Stress-robustness & & & & $\mathrm{x}$ & & & & & & & $x$ \\
\hline Adverse events & & & & & & & & & & & $\ddot{x}$ \\
\hline
\end{tabular}

Fig. 1 Overview of study schedule and main activities. ECG Electrocardiogram, HR Heart rate

any n-3 LCPUFA supplements 3 months prior to the intervention start. Moreover, parents must read and speak Danish in order to be properly informed about the study procedures, and the household should not contain more than five people. Exclusion criteria for the children are serious chronic illnesses and diseases that may interfere with the study outcomes, diagnosed attention-deficit/hyperactivity disorders or other psychiatric illnesses, medication that may affect study outcomes, and concomitant participation in other studies involving dietary supplements or blood sampling. In addition, only one child from each household can participate in the study.

Children of the relevant age and residence will be identified through the Danish Civil Registration System and will be recruited by invitation letter to the parents. Participants may also be recruited through advertisements in Danish newspapers, through networks and social media such as Facebook, and by notices in schools. Parents who express interest will be invited to an informational meeting together with their child, after which they can decide if they wish to participate. If a child is withdrawn from the study, we will ask the parents about the reason for withdrawal. Responding is voluntary, and we will not put pressure on the parents to make them respond.

\section{Randomization, allocation, concealment, and blinding}

Randomization will be performed in blocks of 12 children to ensure approximately equal allocation to the two study groups over the year. A staff member not involved in data collection will produce a computer-generated randomization list with 200 consecutive numbers, each linked to one of the two intervention groups, from which we will produce 200 sealed, sequentially numbered envelopes containing the randomly assigned allocation for that number. The randomization list will be generated from a prespecified seed using the extension package "blockrand" for $\mathrm{R}$ version 3.3.0 [29]. The order in which participants finish the baseline visit will determine their number in the randomization list (and thereby which envelope and allocation they receive). By this procedure, participants and investigators will be blinded to the allocation until the baseline measurement has been completed. However, owing to the nature of the intervention foods, blinding during the intervention is not possible.

\section{Dietary intervention \\ Study foods and instructions}

The fish group will receive oily fish ( $>6 \mathrm{~g}$ fat/100 g) primarily as fresh or frozen fillets of Aquaculture Stewardship Council-certified farmed salmon fillets, but also as cold lunch products such as salmon fish cakes, canned mackerel, marinated herring, smoked trout, and salmon sausages. In order to provide comparable treatments, the control group will receive different cuts of frozen, organic chicken (minced, whole, breast, and thighs) and chicken lunch products such as liver pate, chicken sausages, and chicken meatballs. The participants will be asked to consume $300 \mathrm{~g} /$ week of the intervention products, operationalized as two times per week for dinner and three times per week for lunch. Parents will be asked to substitute some of the fish, poultry, and meat usually consumed by 
the child with the study products and apart from this to maintain the child's usual dietary and physical activity habits during the intervention.

Parents will collect study foods free of charge at NEXS every 1-2 weeks. Fish and poultry for dinner will be provided for the whole family/household, whereas lunch products will be provided for the child only. Parents will also receive a booklet containing inspiration and easy recipes for preparation of study foods and side dishes. Recipes will be matched between the chicken and fish groups with regard to type of dish and energy and fat content.

\section{Safety of study foods}

We have thoroughly considered the safety aspects of the study foods. Two groups of unwanted substances are of special interest in relation to fish intake: methylmercury as well as dioxins and dioxin-like polychlorinated biphenyls (DDPCB). Assuming a weekly intake of $200 \mathrm{~g}$ of farmed salmon, $80 \mathrm{~g}$ of mackerel, and $20 \mathrm{~g}$ of herring, the study fish will provide $8.3 \mu \mathrm{g}$ of methylmercury and $208 \mathrm{pg}$ of DDPCB per week. The tolerable weekly intake (TWI) for these substances (i.e., the estimated amount that can be ingested weekly over a lifetime without appreciable health risk) is $1.3 \mu \mathrm{g} / \mathrm{kg}$ [30] and $14 \mathrm{pg}$ toxic equivalents (TEQ)/kg body weight, respectively [31]. This corresponds to $36 \mu \mathrm{g}$ of methylmercury and 385 pg TEQ DDPCB per week in an average Danish 8-year-old weighing $27.5 \mathrm{~kg}$. Hence, it is estimated that the study fish will provide $23 \%$ and $54 \%$ of the children's TWI for methylmercury and DDPCB, respectively. As fish is the main source of methylmercury in the Danish population [32], it is considered very unlikely that any of the participants will have an intake of methylmercury above the TWI. However, fish contributes only about $30 \%$ of the intake of dioxins and $\mathrm{PCB}$, whereas $70 \%$ is from nonfish sources such as milk, cheese, meat, and fats [32]. The total mean intake of DDPCB among Danish children has been estimated to $0.87 \mathrm{pg} /$ day per kilogram of body weight [32]. An average Danish 8-year-old child is therefore expected to have a DDPCB intake from nonfish sources of $0.61 \mathrm{pg} /$ day per kilogram of body weight, or $118 \mathrm{pg} /$ week. In the general population, there is wide variation in the exposure to DDPCB, caused mainly by the large variation in fish consumption [32]. However, because the families are provided with appropriate amounts of fish products and instructions to meet the intake of $300 \mathrm{~g} /$ week and are not expected to eat markedly more than that, we expect less variation and that their estimated total intake of DDPCB from study fish and nonfish sources will be around $208+118=326$ pg TEQ per week, which is well below the TWI. Also, the families are instructed to replace some of the meat in their diet with the study fish, and therefore the actual mean intake of DDPCB is likely to be lower than calculated. Even though there are individual variations in the intake of DDPCB from sources other than the intervention diet, it is considered unlikely that the children's intake of DDPCB during the intervention will exceed the TWI. The study is thus considered safe.

\section{Measurements \\ Primary outcomes}

Although we are interested to see if oily fish consumption can improve children's overall cardiovascular risk profile, the primary outcomes of the study will be fasting venous plasma triacylglycerol and resting diastolic blood pressure measured in the morning before blood sampling. Local anesthetic patches will be provided to the child to relieve the discomfort of blood sampling. Resting blood pressure will be measured in triplicate by using an automated device with the child lying down after a 10-minute rest, and the mean of the last two measurements will be calculated.

\section{Secondary outcomes}

Cardiovascular health Because the pre-blood sample situation may be stressful for the participants, we will also measure blood pressure during the $3 \mathrm{~h}$ after blood sampling at up to two additional occasions, each time in triplicate and after a 10-minute rest. This step is used to explore whether potential effects of the intervention manifest differently under relaxed and stressful conditions and to link the cardiovascular and behavioral outcomes. Also, diastolic measurements will be supported by systolic blood pressure and heart rate measurements. In addition, children's heart rhythm will be assessed by continuous recording of electrocardiograms using a Holter recording device throughout the 4-h visit at baseline and endpoint. The recordings will be analyzed with respect to heart rate and heart rate variability by Jeppe Hagstrup Christensen, MD, a clinical professor in the Department of Clinical Medicine, Aalborg University Hospital, Aalborg, Denmark. Other secondary outcomes are plasma total cholesterol, lowdensity lipoprotein cholesterol, high-density lipoprotein cholesterol, insulin, glucose, glycated hemoglobin, $\mathrm{C}$-reactive protein, and adipokines.

Cognitive function Cognitive function will be measured after consumption of a standardized breakfast using the following computer-based tests from the Cambridge Neuropsychological Test Automated Battery (CANTAB) [33]: the Motor Screening Test, Reaction Time, Rapid Visual Information Processing, Spatial Working Memory, and Paired Associates Learning. Further, the children 
will perform the d2 Test of Attention in Danish [34], a modified Flanker test [35], and the Stroop test [36] in a Danish version. These are all internationally recognized, validated, standardized tests that assess specific areas of cognitive function with a focus on attention, memory, and executive functions. The tests will be performed by trained project personnel supervised by a psychologist, and to avoid interference by learning effects, the children will pretest the CANTAB tests and the $\mathrm{d} 2$ Test of Attention prior to the baseline examination. The cognitive measurements will be supported by analysis of brain-derived neurotrophic factor (BDNF) in the blood samples. BDNF plays an important role in neuronal survival, and in rodents DHA has been shown to affect BDNF concentrations in brain areas important for attention, executive functions, and memory [37].

Stress robustness At baseline and endpoint, children's physiological stress response will be assessed using a cold pressor task where the child submerges his or her dominant hand in a tub containing cold water for a maximum of 1 minute. We will follow the guidelines of von Baeyer et al. [38] for the use of this task in children, but with the water temperature modified to $5 \pm 2{ }^{\circ} \mathrm{C}$. Children will be instructed that they can remove their hand from the water if they find the test too uncomfortable and will be reminded about this during the test if they display discomfort. Immediately before and after the test, we will measure the child's blood pressure and heart rate, and before and 20 minutes after the test, we will collect a $0.5-$ to $2-\mathrm{ml}$ saliva sample to be analyzed for cortisol concentration. Together with the heart rhythm recorded continuously during the test, these outcomes will be used to assess the child's acute physiological stress response. Finally, a small tuft of hair (approximately 5-20 mg) will be cut from the posterior vertex of the child's head. The $3 \mathrm{~cm}$ of hair located most closely to the scalp will be analyzed for cortisol concentrations, reflecting cortisol and hence stress levels over the last 3 months [39].

Growth and body composition Height, weight, and waist circumference at the level of the umbilicus will be measured by standard procedures, and body mass index z-scores will be calculated. Body composition will be measured by bioimpedance with the child resting in lying position and by triplicate measurements of triceps and subscapularis skinfold thicknesses using a skinfold caliper, and fat mass index and lean mass index will be calculated. We previously found effects of fish oil on the insulin-like growth factor (IGF) axis [40], which stimulates bone and body growth in children, and therefore we will also measure IGF-1, its binding protein, and potentially other growth and bone markers in the blood samples at baseline and endpoint.

\section{Other outcomes}

Behavior Child behavioral and emotional outcomes will be measured by questionnaires to be filled in by the parents, specifically the Strengths and Difficulties Questionnaire [41], the Behavior Rating Inventory of Executive Function questionnaire [42], and the $\mathrm{KINDL}^{\circ}$ quality of life questionnaire developed for healthy children [43]. Parents will receive a short oral instruction in this by trained project personnel supervised by a psychologist. The child version of the KINDL questionnaire will also be administered by interview with the children.

Physical activity and sleep Children's physical activity and sleep will be recorded by using triaxial accelerometers (ActiGraph, Pensacola, FL, USA) during 7 consecutive days and 8 nights prior to the baseline and endpoint visits. Parents will record the times of the child's awakening and falling asleep every day during the recording, and these recordings will be used to define sleep and wake time. Physical activity and sleep will both be seen as outcomes because these behavior-related measures may be affected by the intervention, but they may also be used as covariates because they may affect the primary outcomes.

Compliance Compliance with the oily fish will be assessed as n-3 LCPUFA in erythrocytes, which reflects intake during the last 3-4 months. Also, parents will be asked to record the child's consumption of study fish and poultry on precoded sheets every week during the intervention period and to hand them in when collecting new study foods.

Nutrient biomarkers We will measure serum 25hydroxyvitamin $\mathrm{D}$ at baseline and endpoint because high consumption of oily fish may increase the vitamin D status of the participants. We will also measure serum hemoglobin and ferritin to assess whether the intervention affects iron status (e.g., if the study fish and chicken provided replaces red meat in the diet).

Genetics To assess potential effect modification by genotype, we will analyze polymorphisms in genes coding for proteins involved in the metabolism and signaling of polyunsaturated fatty acids such as the fatty acid desaturase genes, peroxisome proliferator-activated receptor genes, and genes encoding cyclooxygenases [28].

\section{Additional data}

Dietary intake Children's dietary intake will be recorded prior to the baseline and endpoint visits. The parents 
will be required to fill in dietary records of the child's diet for 4 consecutive days, covering 3 weekdays and 1 weekend day, using Internet-based software available for personal computer and smartphone use [44]. Household measures will be used when weighting of the foods is not possible. Additionally, parents will be given a food frequency questionnaire to assess the child's intake of fish, poultry, and other meats.

Background information At baseline, sociodemographic information will be collected and pubertal stage will be evaluated by the child, if necessary aided by a parent, using a self-administered questionnaire on breast development and age at menarche for girls and development of pubic hair for boys. Questionnaires on sun behavior will be administered at both baseline and endpoint.

Adverse events Although we do not expect any adverse events related to the study to occur, parents will be asked to contact us if they experience any adverse events or reactions in their children during the study. At the endpoint visit, we will specifically ask whether they have experienced any adverse events. All reported adverse events will be recorded.

\section{Sample size calculation}

Power calculations were performed using data from a large sample of Danish 8- to 11-year-olds [45]. In order to detect a difference of less than 0.5 SD of the outcomes i.e., $2.7 \mathrm{mmHg}$ in diastolic blood pressure and $0.13 \mathrm{mmol} / \mathrm{L}$ in triacylglycerol between the fish and poultry groups with a significance level of $\alpha=0.05$ and $80 \%$ power, a total of 150 children will need to complete the study. The differences correspond to $4 \%$ and $20 \%$ reductions in blood pressure and triacylglycerol, respectively, which are considered relevant and obtainable. In order to allow for a 15-20\% dropout rate and insufficient blood sampling, we aim to recruit 200 children.

\section{Data analysis considerations}

Outcomes will be analyzed in linear models with adjustment for baseline values, and the difference between the fish and poultry groups will be estimated. All data will be analyzed as completer's analysis (i.e., including only participants who have available data from both baseline and endpoint for a given outcome). For the primary outcomes and associated cardiovascular risk markers, we will also conduct sensitivity analyses. These will include intention-to-treat analysis of all subjects randomized, with imputation of missing values using the baseline observation carried forward method and, if needed, also imputation of missing baseline values. For outcomes where differences are found, we will conduct supportive linear regression analyses exploring potential dose- response associations between erythrocyte n-3 LCPUFA content and outcomes. Secondarily, we will evaluate sex $\times$ treatment interactions. We will also investigate potential effect modification by genotype.

With an increased intake of oily fish or poultry, there is a risk that some children will gain more weight and body fat than expected during normal growth. This may be unevenly distributed between the groups and may impact the cardiovascular outcomes. In that case, we will adjust the outcomes for body mass index or fat mass index to separate the effects of the diets and weight gains. Analysis along the same line of thinking will be performed in case of other unanticipated differences occurring between the two groups.

\section{Discussion}

In most Western countries, official dietary recommendations advocate increased consumption of fish for both children and adults. Recommendations for children are based on the high content of important nutrients in fish, as well as extrapolations from studies in adults where fish and n-3 LCPUFA consumption have been shown to be cardioprotective. However, to underpin such recommendations and get more insight into the public health and biological potential of fish consumption, highquality trials conducted in children are needed. To our knowledge, the FiSK Junior study will be the first randomized controlled trial to investigate the impact of oily fish intake on cardiovascular risk markers in healthy children. Although very few children get lifestyle diseases, elevated cardiovascular risk markers and the metabolic syndrome are seen already in childhood [46]. The Bogalusa Heart Study [47] and the Cardiovascular Risk in Young Finns Study [48] have demonstrated that blood lipid profile and blood pressure show tracking from childhood and adolescence to adulthood. Therefore, small but persistent reductions in risk markers in childhood are likely to be important for long-term cardiovascular risk.

To our knowledge, the FiSK Junior study is also the first randomized trial to investigate effects of oily fish intake on cognitive function and behavior in children attending primary school in a high-income country. The study will provide important knowledge to the fields of fish, n-3 LCPUFA, and cognitive function in childhood, where the overall evidence is inconclusive. The behavioral, emotional, and stress response measurements will provide novel human results that may contribute to filling knowledge gaps on ways to increase mental robustness and prevent mental disorders starting from childhood.

The strengths of the study include the randomized controlled design with fasting blood samples and careful 
characterization of the study population, including measurements of dietary intake, objectively measured physical activity, and genotyping, as well as an objectively measured biomarker of compliance. This intense protocol and frequent consumption of study foods requires willingness and dedication of the participating families, and therefore the expected response rate will be only $1-4 \%$. Thus, the study population will expectedly not be fully representative of Danish children; however, the biological effects of consuming oily fish are not expected to differ between the study population and Danish children in general. The aim of $300 \mathrm{~g} /$ week of fish consumption is within the national recommendation [5], and this amount of oily fish is expected to provide approximately $0.8-1.0 \mathrm{~g} /$ day $\mathrm{n}-3$ LCPUFA, a dose that has been shown to reduce blood pressure and plasma triacylglycerol in previous fish oil trials $[12,14,15]$. Despite our efforts to support children's consumption of study foods by providing bone-free fish as well as poultry free of charge to the whole family, supply commonly eaten lunch products, and provide the parents with cooking tips and easy recipes, we expect that some children will not be able to reach an intake of $300 \mathrm{~g} /$ week. However, compared with previous fish oil trials in children, we still expect to provide a substantial amount of n-3 LCPUFA to the children while balancing what is achievable in a normal diet. Furthermore, this expected variation in children's actual consumption of oily fish will allow us to explore potential dose-response effects of the fish on the many outcomes by use of n-3 LCPUFA in erythrocytes as a measure of oily fish intake during the intervention. Poultry has been chosen as the control food because it is the healthiest alternative and has comparable contents of protein and less n-3 LCPUFA than oily fish.

\section{Conclusions}

The FiSK Junior study will help answer the question "What happens when children eat oily fish?" rather than "How can we make children eat oily fish?" The study will provide novel evidence to be used when setting dietary recommendations for children and thereby help optimize public health starting from childhood.

\section{Trial status}

Recruitment was initiated in August 2016, and the last endpoint examination will be conducted in June 2017.

\section{Additional file}

Additional file 1: Standard Protocol Items: Recommendations for Interventional Trials (SPIRIT) 2013 checklist. (PDF 57 kb)

\section{Abbreviations}

BDNF: Brain-derived neurotrophic factor; CANTAB: Cambridge Neuropsychological Test Automated Battery; DDPCB: Dioxins and dioxin-like polychlorinated biphenyls; DHA: Docosahexaenoic acid (22:6n-3);
ECG: Electrocardiogram; FiSK: Fish, children, health, and cognition; HR: Heart rate; IGF: Insulin-like growth factor; LCPUFA: Long-chain polyunsaturated fatty acids; NEXS: Department of Nutrition, Exercise and Sports, University of Copenhagen; SPIRIT: Standard Protocol Items: Recommendations for Interventional Trials; TEQ: Toxic equivalents; TWI: Tolerable weekly intake

\section{Acknowledgements}

Not applicable.

\section{Funding}

The FiSK Junior study is supported by Nordea-fonden (grant 02-2015-1429). The funder did not have any role in the study design or the decision to publish this protocol. Study foods will be purchased from Skagenfood A/S, Sødam A/S via gaardmester.dk and REMA 1000 Danmark A/S with discount, and provided in kind by Amanda Seafoods A/S.

\section{Availability of supporting data}

Not applicable.

\section{Authors' contributions}

CTD is the principal investigator of the study, conceived of and designed the study, and wrote the first draft of the manuscript. $L L$ conceived of and designed the study and provided critical revisions of the manuscript. $\mathrm{HH}$ helped to design the study, wrote the study protocol, and provided critical revisions of the manuscript. SV helped to design the study and provided critical revisions of the manuscript. MNT helped to design the study and provided critical revisions of the manuscript. CR performed the power calculations and supervised the sections on randomization and statistics. MH supervised and revised the section on the safety of the study foods. JN designed the study, supervised the cognitive measurements, and provided critical revisions of the manuscript. CM conceived of and designed the study and provided critical revisions of the manuscript. All authors read and approved the final manuscript.

\section{Authors' information}

Not applicable.

\section{Competing interests}

The authors declare that they have no competing interests.

\section{Consent for publication}

Not applicable.

\section{Ethics approval and consent to participate}

The study has been approved by the Committee on Biomedical Research Ethics for the Capital Region of Denmark (H-16018225). Informed written consent will be obtained from all custody holders of the children.

\section{Author details}

${ }^{1}$ Department of Nutrition, Exercise and Sports, Faculty of Science, University of Copenhagen, Rolighedsvej 26, 1958 Frederiksberg C, Denmark. ${ }^{2}$ Division of Risk Assessment and Nutrition, National Food Institute, Technical University of Denmark, Søborg, Denmark. ${ }^{3}$ Department of Psychology, Faculty of Social Science, University of Copenhagen, Copenhagen, Denmark. ${ }^{4}$ Centre for Collaborative Health, Aarhus University, Aarhus C, Denmark.

Received: 30 June 2016 Accepted: 7 October 2016

Published online: 21 October 2016

References

1. Delgado-Lista J, Perez-Martinez P, Lopez-Miranda J, Perez-Jimenez F. Long chain omega-3 fatty acids and cardiovascular disease: a systematic review. Br J Nutr. 2012;107 Suppl 2:S201-13.

2. Public Health England. National Diet and Nutrition Survey: Results from Years 1-4 (combined) of the Rolling Programme (2008/2009 - 2011/2012). London: Public Health England; 2014. https://www.gov.uk/government/ uploads/system/uploads/attachment_data/file/310995/NDNS_Y1_to_4_UK_ report.pdf. Accessed 16 Oct 2016.

3. Pedersen A, Christensen T, Matthiessen J, Knudsen VK, Rosenlund-Sørensen M, Biltoft-Jensen A, et al. Danskernes Kostvaner 2011-2013: Hovedresultater. Søborg, Denmark: National Food Institute (DTU); 2015. http://altomkost.dk/ 
fileadmin/user_upload/altomkost.dk/Rapport_Danskernes-Kostvaner-20112013 1_1_pdf. Accessed 16 Oct 2016

4. Commonwealth Scientific Industrial Research Organisation (CSIRO) Preventative Health National Research Flagship, University of South Australia. 2007 Australian National Children's Nutrition and Physical Activity Survey: main findings. Barton, Australia: Commonwealth of Australia; 2008. https://www.health.gov.au/internet/main/publishing.nsf/ Content/8F4516D5FAC0700ACA257BF0001E0109/\$File/childrens-nut-physsurvey.pdf. Accessed 16 Oct 2016.

5. Danish Veterinary and Food Administration. De officielle kostråd - Spis mere fisk. http://altomkost.dk/raad-og-anbefalinger/de-officielle-kostraad/spismere-fisk/. Accessed 3 May 2016.

6. Andersen R, Molgaard C, Skovgaard LT, Brot C, Cashman KD, Chabros E, et al. Teenage girls and elderly women living in northern Europe have low winter vitamin D status. Eur J Clin Nutr. 2005;59:533-41.

7. Braegger C, Campoy C, Colomb V, Decsi T, Domellof M, Fewtrell M, et al. Vitamin D in the healthy European paediatric population. J Pediatr Gastroenterol Nutr. 2013;56(6):692-701.

8. Saggese G, Vierucci F, Boot AM, Czech-Kowalska J, Weber G, Camargo Jr CA, et al. Vitamin $D$ in childhood and adolescence: an expert position statement. Eur J Pediatr. 2015;174:565-76.

9. Leung Yinko SS, Stark KD, Thanassoulis G, Pilote L. Fish consumption and acute coronary syndrome: a meta-analysis. Am J Med. 2014;127:848-57.

10. Miller PE, Van Elswyk M, Alexander DD. Long-chain omega-3 fatty acids eicosapentaenoic acid and docosahexaenoic acid and blood pressure: a meta-analysis of randomized controlled trials. Am J Hypertens. 2014;27:885-96.

11. Mozaffarian D, Geelen A, Brouwer IA, Geleijnse JM, Zock PL, Katan MB. Effect of fish oil on heart rate in humans: a meta-analysis of randomized controlled trials. Circulation. 2005;112:1945-52.

12. Harris WS. Dietary fish oil and blood lipids. Curr Opin Lipidol. 1996;7:3-7.

13. Lauritzen $L$, Christensen JH, Damsgaard CT, Michaelsen KF. The effect of fish oil supplementation on heart rate in healthy danish infants. Pediatr Res. 2008;64:610-4

14. Damsgaard CT, Schack-Nielsen L, Michaelsen KF, Fruekilde MB, Hels O, Lauritzen L. Fish oil affects blood pressure and the plasma lipid profile in healthy Danish infants. J Nutr. 2006;136:94-9.

15. Pedersen MH, Molgaard C, Hellgren LI, Lauritzen L. Effects of fish oil supplementation on markers of the metabolic syndrome. J Pediatr. 2010; 157:395-400.

16. Moore CS, Bryant SP, Mishra GD, Krebs JD, Browning LM, Miller GJ, et al. Oily fish reduces plasma triacylglycerols: a primary prevention study in overweight men and women. Nutrition. 2006;22:1012-24.

17. Ramel A, Martinez JA, Kiely M, Bandarra NM, Thorsdottir I. Moderate consumption of fatty fish reduces diastolic blood pressure in overweight and obese European young adults during energy restriction. Nutrition. 2010;26:168-74.

18. Lauritzen L, Hansen HS, Jørgensen MH, Michaelsen KF. The essentiality of long chain $\mathrm{n}-3$ fatty acids in relation to development and function of the brain and retina. Prog Lipid Res. 2001;40:1-94.

19. Qawasmi A, Landeros-Weisenberger A, Leckman JF, Bloch MH. Meta-analysis of long-chain polyunsaturated fatty acid supplementation of formula and infant cognition. Pediatrics. 2012;129:1141-9.

20. Makrides M, Gibson RA, McPhee AJ, Yelland L, Quinlivan J, Ryan P. Effect of DHA supplementation during pregnancy on maternal depression and neurodevelopment of young children: a randomized controlled trial. JAMA. 2010;304:1675-83.

21. Lauritzen L, Jørgensen MH, Olsen SF, Straarup EM, Michaelsen KF. Maternal fish oil supplementation in lactation: effect on developmental outcome in breast-fed infants. Reprod Nutr Dev. 2005;45:535-47.

22. Lauritzen L, Brambilla P, Mazzocchi A, Harsløf LB, Ciappolino V, Agostoni C. DHA effects in brain development and function. Nutrients. 2016;8:6. do::10 3390/nu8010006.

23. Kennedy DO, Jackson PA, Elliott JM, Scholey AB, Robertson BC, Greer J, et al. Cognitive and mood effects of 8 weeks' supplementation with $400 \mathrm{mg}$ or $1000 \mathrm{mg}$ of the omega-3 essential fatty acid docosahexaenoic acid (DHA) in healthy children aged 10-12 years. Nutr Neurosci. 2009;12:48-56.

24. Kirby A, Woodward A, Jackson S, Wang Y, Crawford MA. Childrens' learning and behaviour and the association with cheek cell polyunsaturated fatty acid levels. Res Dev Disabil. 2010;31:731-42.

25. Smuts CM, Greeff J, Kvalsvig J, Zimmermann MB, Baumgartner J. Long-chain n-3 PUFA supplementation decreases physical activity during class time in iron-deficient South African school children. Br J Nutr. 2015;113:212-24.
26. Asserhoj M, Nehammer S, Matthiessen J, Michaelsen KF, Lauritzen L. Maternal fish oil supplementation during lactation may adversely affect long-term blood pressure, energy intake, and physical activity of 7-year-old boys. J Nutr. 2009:139:298-304.

27. Damsgaard CT, Eidner MB, Stark KD, Hjorth MF, Sjodin A, Andersen MR, et al. Eicosapentaenoic acid and docosahexaenoic acid in whole blood are differentially and sex-specifically associated with cardiometabolic risk markers in 8-11-year-old Danish children. PLoS One. 2014;9:e109368.

28. Minihane AM. Impact of genotype on EPA and DHA status and responsiveness to increased intakes. Nutrients. 2016;8:123. doi:10.3390/nu8030123.

29 R Core Team. R: A language and environment for statistical computing. Vienna: R Foundation for Statistical Computing; 2014. http://www.R-project. org. Acessed 18 Oct 2016.

30. EFSA Panel on Contaminants in the Food Chain (CONTAM). Scientific opinion on the risk for public health related to the presence of mercury and methylmercury in food. EFSA J. 2016;10:2985.

31. Opinion of the Scientific Committee on Food on the risk assessment of dioxins and dioxin-like PCBs in food. Brussels, Belgium: European Commission, Health and Consumer Protection Directorate-General, Scientific Committee on Food; 2001. http://ec.europa.eu/food/fs/sc/scf/out90_en.pdf. Accessed 16 Oct 2016.

32. Pedersen et al. Chemical contaminants 2004-2011. Søborg, Denmark: National Food Institute, Technical University of Denmark; 2013.

33. Cambridge Cognition: cognitive research: CANTAB batteries. Cambridge, UK Cambridge Cognition. http://www.cambridgecognition.com/academic/ cantabsuite/tests. Accessed 27 Sep 2016.

34. Brickenkamp R, Merten T, Hänsgren KD. d2 Test of Attention (Danish version). Virum, Denmark: Hogrefe; 2006.

35. Hillman CH, Pontifex MB, Castelli DM, Khan NA, Raine LB, Scudder MR, et al. Effects of the FITKids randomized controlled trial on executive control and brain function. Pediatrics. 2014;134:e1063-71.

36. Stroop JR. Studies of interference in serial verbal reactions. J Exp Psych. 1935;18:643-61.

37. Rao JS, Ertley RN, Lee HJ, DeMar Jr JC, Arnold JT, Rapoport SI, et al. n-3 Polyunsaturated fatty acid deprivation in rats decreases frontal cortex BDNF via a p38 MAPK-dependent mechanism. Mol Psychiatry. 2007;12:36-46.

38. von Baeyer CL, Piira T, Chambers CT, Trapanotto M, Zeltzer LK. Guidelines for the cold pressor task as an experimental pain stimulus for use with children. J Pain. 2005;6:218-27.

39. Russell E, Koren G, Rieder M, Van Uum S. Hair cortisol as a biological marker of chronic stress: current status, future directions and unanswered questions. Psychoneuroendocrinology. 2012;37:589-601.

40. Damsgaard CT, Harslof LB, Andersen AD, Hellgren LI, Michaelsen KF, Lauritzen L. Fish oil supplementation from 9 to 18 months of age affects the insulin-like growth factor axis in a sex-specific manner in Danish infants. Br J Nutr. 2016:115:782-90

41. Goodman R. The Strengths and Difficulties Questionnaire: a research note. J Child Psychol Psychiatry. 1997:38:581-6.

42. Gioia GA, Isquith PK, Guy SC, Kenworthy L. Behavior Rating Inventory of Executive Function. Child Neuropsychol. 2000;6:235-8.

43. Bullinger M, Brutt AL, Erhart M, Ravens-Sieberer U. Psychometric properties of the KINDL-R questionnaire: results of the BELLA study. Eur Child Adolesc Psychiatry. 2008;17 Suppl 1:125-32.

44. MADLOG. http://www.madlog.dk/da/. Accessed 27 Sep 2016.

45. Damsgaard CT, Dalskov SM, Laursen RP, Ritz C, Hjorth MF, Lauritzen L, et al. Provision of healthy school meals does not affect the metabolic syndrome score in 8-11-year-old children, but reduces cardiometabolic risk markers despite increasing waist circumference. Br J Nutr. 2014;112:1826-36.

46. Johnson WD, Kroon JJ, Greenway FL, Bouchard C, Ryan D, Katzmarzyk PT. Prevalence of risk factors for metabolic syndrome in adolescents: National Health and Nutrition Examination Survey (NHANES), 2001-2006. Arch Pediatr Adolesc Med. 2009;163:371-7.

47. Webber LS, Srinivasan SR, Wattigney WA, Berenson GS. Tracking of serum lipids and lipoproteins from childhood to adulthood: the Bogalusa Heart Study. Am J Epidemiol. 1991;133:884-99.

48. Juhola J, Magnussen CG, Viikari JS, Kähönen M, Hutri-Kähönen N, Jula A, et al. Tracking of serum lipid levels, blood pressure, and body mass index from childhood to adulthood: the Cardiovascular Risk in Young Finns Study. J Pediatr. 2011;159:584-90. 